\title{
Effects of Photoinduced Elastic Responses on Debye-Waller Temperature Measurements
}

\author{
Elisah J. VandenBussche ${ }^{1}$ and David J. Flannigan ${ }^{1}$ \\ ${ }^{1 .}$ Department of Chemical Engineering and Materials Science, University of Minnesota, Minneapolis, \\ USA.
}

The performance and robustness of smaller, faster, and more efficient electronic architectures and devices becomes limited as thermal effects begin to dominate at small scales. The push to shrink dimensionality, and the subsequent impact of thermal effects, has led to the development of methods capable of being used to quantify nanoscale thermal transport and brought about insights into (for example) thermal conductivity, thermoelasticity, and thermal boundary conductance [1]. Owing to the combined nanoscale dimensions and ultrafast timescales associated with thermal-energy carriers, it is useful to extend metrology techniques into these domains in order to directly resolve discrete carrier dynamics. To this end, we are exploring ultrafast electron microscopy (UEM) as a possible method to facilitate access to this parameter space. A typical UEM experiment consists of a pump-probe approach, wherein a specimen is excited (pumped) in situ with a femtosecond laser pulse and subsequently probed with a discrete packet of electrons generated from a photocathode. In this way, the ultrafast structural dynamics of nanoscale and nanostructured specimens can be resolved using imaging and diffraction in the same manner as is done with conventional (static) transmission electron microscopy. Typically, for general ultrafast electron and X-ray scattering experiments, transient temperatures are determined using the Debye-Waller (DW) effect, in which the attenuation of Bragg scattering is related to atomic thermal energies. This approach is appealing, because it provides a direct probe of the lattice response requiring only detection of a scattered electron or X-ray photon, and it can in principle be performed with combined high spatial and temporal resolutions. It has been shown, however, that other factors, in addition to mean atomic displacements, can affect (and even dominate) the intensity of Bragg reflections, most notably thermally-induced specimen tilting and translation [2-5]. Therefore, effects that obfuscate intensity attenuation due to thermal vibrations must be identified, quantified, and deconvoluted in order to accurately and precisely determine nanoscale transient temperatures in this way.

To address this, we are exploring the effects of in situ laser-induced specimen motion (e.g., tilting, bending, translation, etc.) on Bragg-reflection intensities and, from this, developing methods by which associated errors in temperature calculated via the DW effect can be mitigated. More specifically, we have quantified and statistically analyzed the impact of tilting (bending) and translation on DebyeScherrer-ring intensities from polycrystalline aluminum films. Polycrystalline aluminum was chosen because, for a truly random distribution of the full range of orientations in a selected area, the deleterious effects of tilting should be eliminated, and the relatively low-Z (for example, compared to gold) results in a large DW effect for modest temperature changes. Here, parallel-beam diffraction patterns (DPs) were acquired at different tilt angles and from different selected areas, and the ring intensities were quantified, statistically analyzed $(n=5$ measurements per angle/position), and compared. As summarized in Figure 1, it was found that tilting by as little as a fraction of one degree or translating the selected area by as little as $10 \mathrm{~nm}$ produced statistically-significant changes in ring intensity, despite the average grain size (25-nm diameter) being much smaller than the selected area (800- $\mu \mathrm{m}$ diameter). Possible sources of these effects are identified and considered, including changes in 
effective specimen thickness caused by tilting/bending and slight but significant preferential orientation of grains due to surface-energy minimization. Finally, we discuss possible ways to mitigate inaccuracies in DW-calculated temperature due to certain specimen motions, including data normalization and specimen preparation methods [6].

References:

[1] D. G. Cahill et al, Appl. Phys. Rev. 1 (2014), p. 011305.

[2] S. Schäfer et al, Chem. Phys. Lett. 515 (2011), p. 278.

[3] A. O. Er et al, Appl. Phys. Lett. 100 (2012), p. 151910.

[4] D. R. Cremons et al, Ultramicroscopy 161 (2016), p. 10.

[5] R. Li et al, Appl. Phys. Lett. 111 (2017), p. 041105.

[6] This material is based upon work supported by the National Science Foundation Graduate Research Fellowship Program under Grant No. 00039202 and by the Arnold and Mabel Beckman Foundation in the form of a Beckman Young Investigator Award.
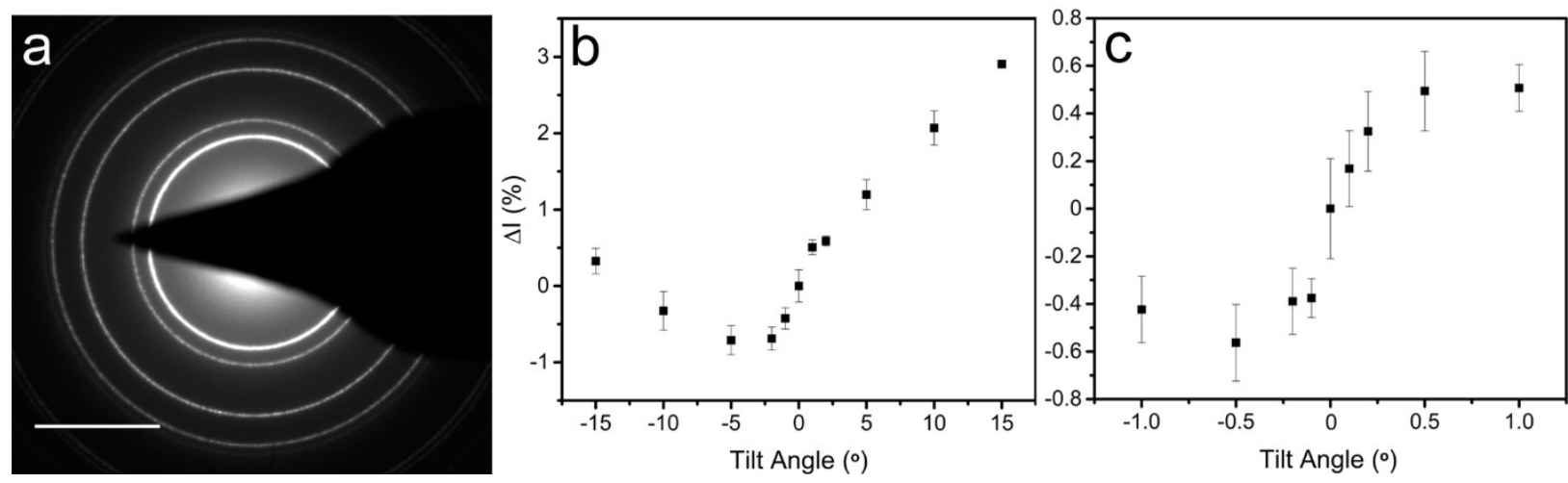

Figure 1. (a) Representative diffraction pattern (DP) from polycrystalline aluminum. Scale bar $=5 \mathrm{~nm}^{-1}$. (b) Relative percent change in intensity of the 220 Debye-Scherrer ring versus alpha-tilt angle determined from DPs acquired with a camera length of $250 \mathrm{~mm}$ at $200 \mathrm{kV}$ and with an $800-\mu \mathrm{m}$ selectedarea aperture. All measurements were performed in random order. The error bars represent one standard deviation of five measurements at each tilt angle. (c) The same experiment as in (b) but over a smaller range of tilt angles. 